\title{
Euler-Vorlesung in Sanssouci
}

\section{von Reinhard Bölling}

Im November 1992 faßte das Organisationskomitee „Berliner Mathematisches Kolloquium“ den Beschluß, jährlich einmal als „außergewöhnliches Ereignis“ eine gemeinsame Veranstaltung der mathematischen Institute Berlins und Potsdams durchzuführen. Die zugrundeliegende Idee war, den Chancen und Möglichkeiten, die nach dem Fortfall der Berliner Mauer in der nunmehr vorhandenen großen Konzentration an institutionalisierter Mathematik im Berliner Raum lagen, Rechnung tragen zu wollen. Im Mittelpunkt sollte ein Gastvortrag stehen, der von einer weltweit anerkannten Forscherpersönlichkeit zu einem Thema von allgemeinerem Interesse vor einem mathematisch gebildeten aber nicht notwendig aus Spezialisten bestehenden Publikum zu halten ist. Da die Biographie eines der hervorragendsten Mathematiker aller Zeiten, Leonhard Euler, eng mit Berlin und Potsdam verbunden ist (einem Ruf Friedrichs II folgend kam Euler 1741 nach Berlin, wo er 25 Jahre wirkte), sollte die Veranstaltung den Namen „Euler-Vorlesung“ tragen. In Potsdam bot sich die Möglichkeit, die EulerVorlesung im Schloßtheater von Sanssouci als ausgesprochen repräsentativem Ort stattfinden zu lassen, dessen imposante Kulisse den Wunsch nach festlicher Umrahmung in ganz besonderer Weise in Erfüllung gehen ließ.

Die Auswahl des Vortragenden obliegt einer unabhängigen Jury aus namhaften Mathematikern, der gegenwärtig M. Grötschel (Berlin), F. Hirzebruch (Bonn) und K.-H. Hoffmann (München) angehören. Die Jury entscheidet selbst über den Nachfolger bei Ausscheiden eines Mitgliedes.

Zum Programm der „Euler-Vorlesung" gehört auch die Vorstellung des eingeladenen Gastes in einer Laudatio. Eingeleitet wird die Veranstaltung durch einen historisch orientierten Vortrag. Zur festlichen Ausgestaltung tragen musikalische Darbietungen von Studenten und Lehrkräften des Instituts für Musik und Musikpädagogik der Universität Potsdam bei.

Der Minister für Wissenschaft, Forschung und Kultur des Landes Brandenburg ist Schirmherr der „Euler-Vorlesung in Sanssouci“. Ein Vertreter des Ministeriums sowie der Rektor (oder ein Vertreter) der Universität Potsdam richten zur Eröffnung Grußworte an die Versammlung. Durch ein professionell gestaltetes Plakat, gedruckte Einladungen und Pressemitteilungen soll eine größere Wirkung nach außen erreicht werden.

Die Kosten der Veranstaltung werden von den sechs beteiligten Institutionen (die Fachbereiche Mathematik der drei Berliner Universitäten und der Universität Potsdam, das Konrad-Zuse-Zentrum für Informationstechnik und das Weierstraß-Institut für Angewandte Analysis und Stochastik (beide Berlin)) sowie dem Ministerium für Wissenschaft, Forschung und Kultur des Landes Brandenburg unter Einbeziehung von Sponsoren gemeinsam getragen. Die Organisation vor Ort wird vom Institut für Mathematik der Universität Potsdam mit Unterstützung weiterer dort vorhandener universitärer Einrichtungen geleistet.

Es ist geplant, die Euler-Vorlesung jedes Jahr im Mai (möglichst am 3. Freitag) stattfinden zu lassen.

Nachfolgend eine Übersicht über die bisher gehaltenen Vorträge:
1. Euler-Vorlesung am 18. Mai 1993

R. Bölling (Potsdam): Leonhard Euler - aus der Zeit seines Wirkens in Berlin

B.-W. Schulze (Potsdam): Laudatio auf Raoul H. Bott

R. Bott (Harvard University): On Invariants of Manifolds

2. Euler-Vorlesung am 20. Mai 1994

H.-C. Im Hof (Basel): Geschichte und aktueller Stand der Euler-Ausgabe

F. Hirzebruch (Bonn): Laudatio auf Roger Penrose

R. Penrose (Oxford): The complex Structure of the Universe

Bei beiden Veranstaltungen war das Schloßtheater bis auf den letzten Platz gefüllt, wobei erfreulicherweise auch viele Studenten dem Auditorium angehörten.

Die Euler-Vorlesung 1995 findet am 19. Mai mit folgenden Vorträgen statt:

E. Knobloch (Berlin): Das große Spargesetz der Natur: Zur Tragikomödie zwischen Euler, Voltaire und Maupertius

F. Hirzebruch (Bonn): Laudatio auf Armand Borel

A. Borel (Princeton): Zeta Functions at Integers in Analysis and Topology

Alle Interessenten sind herzlich eingeladen (die Veranstaltung beginnt um $14 \mathrm{Uhr}$ im Schloßtheater von Sanssouci).

Adresse des Autors:

Prof. Dr. R. Bölling

Universität Potsdam

Institut für Mathematik

Postfach 601553

D-14415 Potsdam. 